\title{
Die These: Corona lehrt uns, dass (Unternehmens-)Netzwerke auch virtuell gut funktionieren
}

\begin{abstract}
„Nein“, sagt Dr. Markus Neufeld, Projektleiter von CREAPOLIS, der Innovations- und Vernetzungsplattform der Hochschule Coburg. Seine Überzeugung:
\end{abstract}

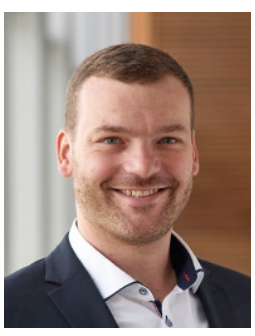

Dr. Markus Neufeld
„Wir vernetzen Menschen innerhalb und außerhalb der Hochschule miteinander, persönlich und institutionell, lokal und regional. Wir nennen das , connect ${ }^{\text {- }}$ - das ist Teil unserer Mission. Und wir sind davon überzeugt, dass es auch zukünftig reale Orte braucht, um unsere Mission zu erfüllen. Warum?

Atmosphäre und Emotion: Gerade wenn es um, entertainige' Formate geht wie Slams oder Pitches, die im Wissenstransfer zunehmend Eingang finden, spielen die Atmosphäre, die durch den Ort entsteht - beispielsweise eine Gewerbebrache -, das Licht und die Musik eine zentrale Rolle. Die Veranstaltung wird zum Erlebnis, das in Erinnerung bleibt und viele Sinne anspricht. Eine Vortragsveranstaltung funktioniert auch per Stream auf die Couch. Eine laue Sommernacht, der Duft von Bratwürsten oder gegrilltem Gemüse und die Illumination von Industriearchitektur lassen sich hingegen nur schwer digital transportieren.

Gleichzeitigkeit und Zufälligkeit: Treffpunkte wie unser Makerspace (eine offene Werkstatt) oder die genannten Events zeichnen sich dadurch aus, dass Menschen zufällig bzw. gleichzeitig am gleichen Ort sind und sich begegnen können. Aus der Innenarchitektur weiß man, dass es für Kreativität und Innovation Raum für zufällige Begegnungen braucht. Wir haben so einen Raum. Und wir wollen Begegnung ermöglichen. Ja, es gibt die Funktion des ,Breakout Rooms ' in den diversen virtuellen Anwendungen. Aber das ist wirklich nicht das gleiche.

Digitaler Overkill: Mich strengt eine Videokonferenz oft mehr an als ein reales Meeting. Der Fokus ist hoch, die Ablenkung gefühlt geringer. Und ich bin froh, wenn ich dann mal den Laptop runterklappen kann. Mir ist die Bildschirmzeit - nicht nur bei Kindern - gerade zu viel. Der Zwang zur Digitalisierung wird zum Overkill. Deshalb sind, nein wären reale Treffen bei einer Tasse Kaffee oder einem fränkischen Bier gerade eine willkommene Abwechslung.“

„Ja“", meint Hendrik Montag-Schwappacher, Geschäftsführer des Innovations-Zentrums Region Kronach (IZK). Der Verein will die Innovationskraft regionaler Unternehmen stärken und Brücken in die Zukunft bauen:

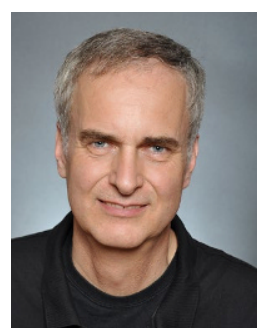

Hendrik MontagSchwappacher
„Gemeinschaften leben von Begegnung. Wenn Menschen zusammenkommen, entstehen gemeinsame Interessen, wird zu Zusammenarbeit angeregt und das Netzwerk lebt. Dabei werden Veranstaltungen auch vom Zufallselement der Gespräche am Stehtisch getragen. Dieses Zufallselement entfällt bei Onlineveranstaltungen, aber vielleicht funktioniert das Netzwerken ja auch online?

$\mathrm{Zu}$ Beginn des Corona-Lockdowns im März 2020 wurde klar, wie sehr Unternehmen unter den Schutzmaßnahmen leiden. Angebote für unternehmensrelevante Informationen kamen flott aus dem Netzwerk und suchten ein passendes Forum. Das neue Format des IZK Cyber Dienstag wurde zur Onlineplattform für coronabedingte Maßnahmen.

Der aktuell größte Vorteil einer Videokonferenz: Wer sich online trifft, kann kein Corona übertragen. Aber auch der zweite Vorteil wurde schnell deutlich: Eine Videokonferenz erfordert keine Anreise und kann daher im Tagesablauf anders positioniert werden. Der IZK-Cyber Dienstag findet regelmäBig von 10.30 bis $12.00 \mathrm{Uhr}$ statt und passt in viele Kalender. Die einfachere Organisation der Veranstaltung führt zu einer raschen Umsetzung; das Wissen Einzelner wird schnell zum Wissen der Gemeinschaft. Hinzu kommt eine andere Intensität der Veranstaltung. Die Bedingungen erfordern hohe Disziplin der Teilnehmer, weil z.B. nicht zwei Personen gleichzeitig sprechen können. Der Austausch von Informationen bekommt eine Intensität, die in konventionellen Sitzungen nicht immer erreicht wird. Hinzu kommt: Ein Chatfenster hilft dabei, während eines Vortrags Fragen zu stellen. Diskussionen werden besser steuerbar und erhalten mitunter eine beachtliche inhaltliche Tiefe.

Die Corona-Krise hat Videokonferenzsysteme aus der NerdNische geholt und zur Alltagstechnologie geadelt. Nun gilt es, diese Potenziale bei Kundenakquise, Projektmanagement und Personalrekrutierung zu heben. Potenziale, die unsere Unternehmen nach Corona dringend brauchen.“ 